\title{
REDIMAT
}

Journal of Research in Mathematics Education

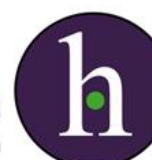

Instructions for authors, subscriptions and further details:

http://redimat.hipatiapress.com

\section{Encyclopedia of Mathematics Education.}

Michèle Artigue ${ }^{1}$

1) Université de Paris Diderot - Paris 7, Francia.

Date of publication: Febrero $24^{\text {th }}, 2016$

Edition period: Febrero 2016-Junio 2016

To cite this article: Artigue, M. (2015). Task Design in Mathematics Education. An ICMI study 22. REDIMAT, 5(1), 104-106. doi: 10.4471/redimat.2016.1975

To link this article: http://dx.doi.org/10.4471/redimat.2016.1975

\section{PLEASE SCROLL DOWN FOR ARTICLE}

The terms and conditions of use are related to the Open Journal System and to Creative Commons Attribution License (CC-BY). 
REDIMAT, Vol. 5 No. 1 February $24^{\text {th }} 2016$ pp. 104-106

\section{Review}

Watson, A. \& Ohtani, M. (Ed.) (2015). Task Design in Mathematics

Education. An ICMI study 22. Netherlands: Springer.

1 libro titulado "Task Design in Mathematics Education »,
asociado al estudio ICMI 22, resulta de un proceso colectivo común
a los estudios ICMI: preparación de un documento de discusión por el IPC (Comité Internacional del Programa) y llamada a contribuciones, organización de la conferencia asociada al estudio, producción de una obra final apoyándose en las aportaciones de la conferencia, y particularmente de sus grupos de trabajo temáticos. Este proceso se refleja en la estructura del libro. Empieza con un capítulo introductorio en el que Anne Watson y Minoru Ohtani, los dos co-editores, ofrecen una visión sintética del estudio antes de terminar con comentarios más personales sobre el trabajo realizado, las perspectivas y necesidades de investigación que se derivan. Los siguientes cinco capítulos con varios autores, reflejan el trabajo colectivo sobre los temas que estructuraron el estudio, reformulados en los siguientes ejes: "Frameworks and principles piloting task design", "The relationships between task design, anticipated pedagogies and student learning", "Accounting for student perspectives in task design", "Design issues related to text-based tasks" y "Designing mathematics tasks: the role of tools". A esto le siguen cuatro nuevos capítulos que están asociados a las plenarias invitadas a la conferencia, escritos respectivamente por Michal Yerushalmy, Berta Barquero y Mariana Bosch, Toshiakira Fujii, y Jan de Lange. El libro se cierra con dos capítulos de comentarios y reflexiones de Ken Ruthven y yo misma, componiendo una obra de 339 páginas.

El capítulo 2 se dedica a los marcos y principios del "diseño de tareas". Se trata del más importante del libro, con 64 páginas. A través de un estudio retrospectivo, nos muestra que la concepción de tareas, su organización en secuencias, su escenificación didáctica en el aula, su adaptación a distintos contextos, han siempre estado en el corazón de la investigación en didáctica de las matemáticas. El lector se afronta aquí a la diversidad de enfoques en 
los cuales se desarrollan los trabajos sobre estas cuestiones, a la complejidad del paisaje resultante. Para organizarlo, los autores introducen interesantes distinciones, como por ejemplo, las entre "diseño como intencion" y "diseño como implementación", o entre "teorías como recurso" o "teorías como producto". Se interrogan las relaciones que existen entre principios de diseño y marcos teóricos, se pregunta hasta qué punto principios y teorías guían efectivamente el diseño y cuál es la parte de artesanía o arte en el. Múltiples ejemplos ayudan el lector a dar sentido a las distinciones introducidas y percibir la diversidad de respuestas que se ofrecen a las cuestiones planteadas, haciendo de este capítulo una pieza clave del libro.

La obra continua por capítulos que abordan sucesivamente el diseño de tareas desde distintos ángulos complementarios : concepción de tareas, su interpretación y transformación por sus utilizadores, profesores como estudiantes; potencialidades y límites de las "tareas basadas en texto", desde aquellas que se encuentran clásicamente en los libros de texto a las que se proponen en la actualidad usando una diversidad de recursos tecnológicos; influencia de los avances tecnológicos. La impresión de diversidad de las cuestiones estudiadas, de enfoques, de trabajos, se refuerza, a pesar de los esfuerzos indiscutibles de los autores de los distintos capítulos para organizar esta diversidad.

Con los cuatro capítulos asociados a las conferencias plenarias, se focaliza la reflexión. Michal Yerushalmi pone en evidencia, a través del análisis del diseño de un manual electrónico sobre funciones, las profundas transformaciones del libro de texto generadas por la cultura numérica; Berta Barquero, Marianna Bosch, y Toshiakira Fujii permiten entender mejor dos fuertes tradiciones de diseño, por un lado, aquella que se ha constituido alrededor de la teoría de situaciones didácticas y del concepto de ingeniería didáctica, y extendido en el marco de la teoría antropológica de lo didáctico, por otra parte, la de las "lesson studies". Jan de Lange, por su parte, a la luz de su experiencia en el Instituto Freudenthal, reivindica una concepción del diseño como artesania y arte, y cuestiona el interés de quererlo sistematizar o codificar.

Como lo escribí en la conclusión de mi capítulo de comentarios, esta obra ofrece sin ninguna duda una buena panorámica del estado del arte sobre el diseño de tareas en educación matemática, y como tal, es de gran valor. Se muestra bien el carácter esencial de las cuestiones que se trabajan 
en este dominio, la riqueza, la diversidad y la creatividad de las investigaciones realizadas. Se proponen numerosas herramientas para organizar este campo de estudio, y se ofrecen numerosos ejemplos inspiradores. Sin embargo también cabe destacar que la lectura de esta obra muestra cómo queda mucho por hacer para consolidar los conocimientos y dar más coherencia a un paisaje todavía fragmentado.

Michèle Artigue, Université de Paris Diderot - Paris 7, Francia michele.artigue@univ-paris-diderot.fr 